\title{
HACER CIENCIA AL SUR DEL MUNDO
}

\author{
Ximena Azúa Ríos
}




\section{XIMENA AZÚA RÍOS}

Es Licenciada en Lengua y Literatura Hispánicas, Magíster en Estudios Latinoamericanos y Doctora en Literatura de la Universidad de Chile. Académica de la Facultad de Ciencias de la Universidad de Chile. Se desempeñó como directora del Departamento de Educación de la Facultad de Ciencias Sociales de esta misma Universidad y como coordinadora de programas tanto de investigación como de asuntos académicos. 


\section{HACER CIENCIA AL SUR DEL MUNDO}

\section{"Solo la vida puede conocer la vida" Francisco Varela}

La de los años sesenta fue una década intensa, convulsionada -qué duda cabe-, pero por sobre todo de una explosión de creatividad, de energía y de modernidad en nuestro país. A la reforma agraria, la reforma educacional, la ampliación de la matrícula en todos los niveles educacionales, en particular en la Universidad de Chile, se sumó la creación de los Colegios Regionales de Temuco, la Serena y más, que daría origen a las sedes regionales de la Universidad.

En la Universidad de Chile existía un puñado de académicos de distintas facultades, fundamentalmente de Medicina, Ciencias Físicas y Matemáticas y en menor medida del Instituto Pedagógico, entre otras, pero también de diversa formación veterinaria, químicos y farmacéuticos, odontólogos, etc., inquietos y deseosos de realizar ciencia básica en nuestro país. Ese sueño tenía larga data (en los años veinte el rector de la época, Carlos Charlin, presentó al Consejo Universitario una propuesta de creación de una Facultad de Ciencias Naturales y Matemáticas), sin embargo, fue en la década de los sesenta donde se materializó esta anhelada y ambiciosa idea.

Inicialmente se creó el Instituto de Ciencias a través de un Decreto Universitario del 30 de noviembre de 1962, que implicó un paso hacia el objetivo común de los científicos. Más tarde, en sesiones del 16, 23 y 30 de diciembre de 1964, el Consejo Universitario acordó la creación de la Facultad de Ciencias, fijó sus objetivos fundamentales y constituyó su claustro inicial. El Supremo Gobierno prestó su aprobación a ese importantísimo acuerdo del Consejo Universitario por decreto del Ministerio de Educación Pública No 135 del 14 de enero de 1965. La Facultad de Ciencias es la única unidad académica de la Universidad de Chile creada de esa manera, pues respondía a un doble anhelo: por una parte, de los académicos que deseaban desarrollar ciencia en el país y, por otra, de las autoridades políticas de la época de asentar las bases del desarrollo científico nacional.

Los objetivos asignados a la décimo tercera facultad de la Universidad de Chile fueron los siguientes: 
a. Desarrollar, sin perjuicio de las que se efectúen en otras facultades, investigaciones que tiendan esencialmente a la ampliación del conocimiento en el campo de las ciencias matemáticas y naturales;

b. Elaborar y aplicar los planes de estudios de las licenciaturas y doctorados en ciencias matemáticas y naturales. Estos planes podrán cumplirse en cursos propios de la Facultad de Ciencias y en cursos que funcionen en otras facultades;

c. Organizar cursos y actividades especiales de perfeccionamiento en las ciencias matemáticas y naturales para graduados y personal de la docencia superior, y

d. Coordinar, a través de sus miembros que lo sean también de otras facultades, la investigación y la enseñanza de las ciencias básicas en la Universidad.

El decreto incluía el nombramiento, por un año, de 26 profesores $^{1}$, la mayoría de los cuales cumplían con lo que era condición imprescindible, vale decir, publicar en el área de su competencia. La designación de esos profesores era ad honórem y su tarea era elegir a los 10 profesores titulares definitivos con los que empezaría la nueva facultad en sus tareas regulares.

Los primeros académicos de la Facultad de Ciencias fueron las mismas personas que se reunieron a planificar las acciones para lograr su creación; su primer Decano, el Dr. Gustavo Hoecker Salas, fue quien llevó adelante sus cimientos. Sin embargo, una tarea de esta envergadura requería de la colaboración de muchos, entre ellos de algunos que no pertenecían a nuestra Universidad. Fue así que se contó con la colaboración del Dr. Mario Ricardi de la Universidad de Concepción; y los profesores de la Pontificia Universidad Católica, Dres. Joaquín Luco y Héctor Croxatto, a quienes se les designó profesores honorarios de la facultad posteriormente.

La facultad era producto de esos años sesenta, creativos y colaborativos, y por sobre todo generosos en sus sueños. De allí la participación de distintos académicos de otras universidades del país. También se nutrió de investigadores de otros lares. Así, por ejemplo, tras el golpe militar en Argentina, en 1966, se intervino la Universidad de Buenos Aires y todos sus académicos renunciaron para solidarizar con las autoridades que fueron desplazadas. Entonces buscó nuevos rumbos en

1 La nómina estaba integrada por César Abuauad Abujatum, Gabriel Alvial Cáceres, Carlos Andrade Plaza, Mitzy Canessa, Claudio Anguita Cáceres, Arturo Arias Suárez, Danko Brncic Yuricic, Sergio Bunel Oyanedel, Julio Cabello Ruz, Osvaldo Cori Moully, Héctor Croxatto Rezzio, Francesco Di Castri Liviero, Humberto Fuenzalida Villegas, Roberto González Rodríguez, Carlos Grandjot Reins, Jorge Hodgson Gibson, Gustavo Hoecker Salas, José Luis Izquierdo Fernández, Nahum Joel Sobelmann, Edgard Kausel Vecchiola, Jochen Kummerow Schuelke, Humberto Maturana Romecín, Dario Moreno Osorio, Jorge Muñoz Cristi, Hermann Niemeyer Fernández, Igor Saavedra Gativa y Félix Schwartzmann Turkenich. 
el extranjero un número importante de los docentes de la Facultad de Ciencias Exactas y Naturales de dicha universidad, quienes se vinieron a nuestro país para incorporarse a la Facultad de Ciencias de la Universidad de Chile.

Juan Gómez Millas -Ministro de Educación en esa época- vio en ese conflicto la oportunidad de sumar científicos latinoamericanos a esa naciente facultad y con ellos creó nuevos grupos de trabajo. Dos de los científicos argentinos que se incorporaron en esa ocasión y que se mantienen activos hasta hoy en la facultad pertenecen al Departamento de Química. Se trata de los Profesores Irma Crivelli y Bruce Cassels.

¿Qué hace querer a un número pequeño de individuos ser científicos en un país pobre, con una escasa tradición científica? El fin principal del cuerpo académico que recién se conformaba fue hacer buena investigación para hacer buena docencia; formar científicos y formar académicos de otras universidades. En este sentido, la Facultad de Ciencias ha tenido un papel importante en la generación de académicos e investigadores a lo largo de Chile. La facultad se creó el año 65 y con ello comenzó la febril actividad de armarse como tal, fortaleciendo su cuerpo académico, sus instalaciones y posteriormente los programas académicos: licenciaturas, programas de posgrados y más tarde las carreras profesionales.

La creación de cualquier facultad implica una inversión importante en infraestructura, más aún si hablamos de una Facultad de Ciencias. En el caso de esta ha sido un camino largo y pedregoso llegar a tener instalaciones acordes con los estándares internacionales. En el año 1968, en terrenos adquiridos por la Universidad de Chile a la chacra Santa Julia, se inició la construcción de instalaciones provisorias para el funcionamiento de la facultad en un recinto propio. Con casi 800 metros cuadrados de una precaria infraestructura comenzaron sus funciones provisoriamente algunos biólogos y químicos. Este fue el inicio de las emblemáticas construcciones conocidas como "las barracas", que aún cobijan a muchos científicos después de 50 años.

Estos pabellones de madera, símbolo de una época de importantes cambios en el país, no solo han perdurado en el tiempo sino que han sido testigos privilegiados de las numerosas investigaciones que se han realizado, de la formación de muchos científicos para el país y del crecimiento alcanzado por la facultad para transformarse en uno de los principales centros de investigación ${ }^{2}$.

2 Algunos datos que sustentan esta afirmación: en el año 2013 la facultad tenía 95 proyectos FONDECYT Regular y de Iniciación vigentes, equivalentes al 16,5\% de los proyectos vigentes de la Universidad de Chile. Si se normaliza esta cifra de acuerdo al número de académicos con dedicación igual o superior a 22 horas, se obtiene que la Facultad de Ciencias posee un factor de 0,84 proyectos vigentes por académico en relación al promedio de la Universidad de Chile, 
Las barracas, junto al ciclotrón, representan los dos elementos tangibles que marcaron la colonización de los terrenos de la chacra de Santa Julia. Las primeras sentaron las bases de la infraestructura, sin importar la forma, para la generación de nuevos conocimientos, y el segundo implicó un hito en el desarrollo de la investigación en física nuclear.

El ciclotrón era un acelerador de partículas que llegó desde Estados Unidos en el marco de la política de la Alianza para el Progreso con el proyecto de cooperación interuniversitaria, conocido como Convenio Chile-California y su establecimiento a través de la Fundación Ford. Su llegada, llena de expectativas, marcó un hito en el desarrollo de la Facultad de Ciencias e implicó una presencia física en el nuevo campus que congregó a científicos y estudiantes. Su instalación y mantenimiento requirieron esfuerzos y gran creatividad, pues se carecía de los repuestos originales y el acelerador venía ya con un cierto desgaste. A pesar de ello, mientras estuvo en funcionamiento se realizaron variados y exitosos experimentos e investigaciones desarrolladas por profesores y estudiantes de física.

El ciclotrón no solo marcaba una presencia física en la nueva territorialidad de la facultad, sino que materializaba un trabajo disciplinario entre las ciencias naturales y exactas. En palabras del decano Gustavo Hoecker Salas:

"Podría decirse que este surgimiento del interés en la física nucleary el empleo de isotopos trajo consecuencias más amplias: los biólogos y quimicos se encontraron con los físicos y matemáticos y aprendieron, por una parte, a superar desconfianzas y cooperar en investigación en curso o nuevas y, por otra, a tomar conciencia de la necesidad de formar urgentemente en Chile una nueva generación de científicos de un modo más sistemático que aquel más casual por medio del cual llegaron ellos a la investigación".

A la vez que se iba dando una primera fisonomía a lo que hoy conocemos como el Campus Juan Gómez Millas, también se ocupaban espacios extramuros y fuera de la ciudad que daban impulso a la investigación, como el Laboratorio de Montemar y la Estación de Hidrobiología en Rapel.

que es 0,27 proyectos por académico. Otro elemento a considerar son los artículos publicados en el año 2013 en revistas ISI. Este índice, normalizado por el número de académicos con dedicación igual o superior a 22 horas, fue de 2,17 artículos por académico en la Facultad y en la Universidad de Chile fue 0,73 artículos por académico. Fuente: Anuario Facultad de Ciencias 2013.

3 Discurso inaugural del Decano Gustavo Hoecker Salas en 1967, con motivo de la llegada del ciclotrón. 
En la zona conocida como Montemar, en la Región de Valparaíso, está ubicada la casa que alberga desde 1965 al Laboratorio de Fisiología Celular fundado por el Profesor Mario Luxoro. Este funcionaba desde comienzos de los sesenta, justo en frente de su ubicación definitiva en las dependencias de la entonces sede de Valparaíso de la Universidad de Chile. En el año 1965 la casa fue comprada por la misma Universidad para alojar en forma exclusiva a este laboratorio. El ritmo, la independencia y la metodología de trabajo que Montemar desarrollaba requerían un espacio independiente que permitiera desplegar la energía y dinamismo con que se trabajaba.

El Profesor Luxoro había comenzado a investigar sobre la transmisión de impulsos eléctricos en calamares en su estadía en Estados Unidos y retornó a Chile con el propósito de continuar y profundizar este trabajo pensando en la posibilidad que el Océano Pacífico chileno le podía dar a través de la jibia, el calamar gigante que habita estas aguas. La existencia de este enorme calamar generaba incredulidad en los colegas científicos con quienes trabajaba Luxoro en Estados Unidos. Pero sí, efectivamente la jibia existía y era gigante, así como su axón, que es la prolongación de la neurona que conduce el impulso nervioso. Las dimensiones y la gran disponibilidad de jibia en ese período permitieron realizar avances importantísimos relacionados con la trasmisión del impulso eléctrico y del transporte de iones en las células nerviosas, que tienen validez para todas las especies. Los descubrimientos realizados en Montemar le dieron a este laboratorio renombre científico mundial.

La casa que acogió al laboratorio se transformó en una escuela privilegiada, un espacio de trabajo integral que permitía y exigía desarrollar nuevas ideas y nuevas soluciones; los propios investigadores aprendían a diseñar instrumentos específicos para desarrollar sus trabajos en los talleres mecánicos y eléctricos que se habían habilitado. Todo esto sucedía en forma natural, como parte del quehacer particular del trabajo científico ${ }^{4}$.

En 1973 vino el golpe de estado y entre muchas de las consecuencias que trajo para el país, la Universidad de Chile y la Facultad de Ciencias, produjo una diáspora de científicos. La jibia, por su parte, producto de corrientes frías, desapareció. La actividad de Montemar bajó, pero se mantuvo con nuevas investigaciones acerca de la musculatura del picoroco. Hoy en día en el Laboratorio de Montemar se realizan

4 En Montemar trabajaron destacados investigadores chilenos. Entre los pioneros estuvieron Eduardo Rojas, Alejandro Cintolessi, Mitzy Canessa, Fernando Vargas y Sigmund Fisher. Luego vinieron Cecilia Hidalgo, Ramón Latorre, Pedro Verdugo, Francisco Bezanilla, Julio Vergara, Cecilia Vergara y Juan Bacigalupo, entre otros. También llegaron científicos extranjeros de la talla de Richard Keynes y Clay Armstrong, atraídos por los aportes de primer nivel que se realizaban en este laboratorio. 
experimentos acotados. Hay menos movimiento en el que ayer fuera un fulgurante centro irradiador de conocimiento, pero las jibias han vuelto y Montemar sigue allí.

La Estación de Hidrobiología, por su parte, ubicada a orillas del lago Rapel, es el lugar en el que los académicos e investigadores del Departamento de Ciencias Ecológicas han desarrollado importantes líneas de investigación, principalmente, en el área de la limnología. Esta área se desarrolló durante la década de 1970, primero en el Departamento de Biología de la facultad, y fue considerada -más tarde- como grupo de trabajo al crearse el Departamento de Ciencias Ecológicas en el año 1983. Los trabajos de este grupo se iniciaron el año 1975 analizando la calidad del agua del embalse Rapel, el cual se había comenzado a llenar a fines del año 1968. Facilitó este trabajo la donación de una casona y terrenos a orillas del lago Rapel por parte de ENDESA a la Universidad de Chile ${ }^{5}$. La Profesora Irma Vila y su equipo recorrían los $40 \mathrm{~km}$ desde los ríos hasta la represa en una lancha Saeta, no sin extraviarse a veces en las ensenadas y llegar a la estación a remo o arrastrando el bote por fallas en el motor. Las lluvias, el frío invernal y el calor del verano con los infaltables y abundantes zancudos, no impidieron el desarrollo de las primeras tesis y consiguientes publicaciones ${ }^{6}$. En la actualidad siguen desarrollando investigaciones y contribuyendo a la formación de los estudiantes de pregrado y posgrado.

\section{DE LA FORMACIÓN DE CIENTÍFICOS Y CIENTÍFICAS PARA CHILE A LA ALFABETIZACIÓN CIENTÍFICA DE LA SOCIEDAD}

La Facultad de Ciencias se creó con un norte claro: hacer ciencia formando científicos y científicas para el país. Pero ¿cómo se formarían estos científicos? ¿Cómo aprenderían y para qué? ¿Cuál sería la orientación de las asignaturas? ¿Cómo se combinaría la investigación con la docencia? Las respuestas a estas preguntas se plasmaron en lo que hoy se recuerda como el Libro Naranja, conocido así por el color de su portada.

Algunos pasajes de su prólogo que aquí atraemos tienen una vigencia que sorprende, pero no nos deben llevar a engaño; su actualidad dice relación con la concepción de enseñanza de aprendizaje activo y dinámico participativo que se concibe con el aprender haciendo:

5 Capítulo IV. Limnología: la vida en el agua, la teoría y la práctica. En: Montecino V, Orlando J (eds). Ciencias Ecológicas 1983-2013: Treinta años de investigaciones chilenas. Editorial Universitaria. Santiago, Chile Vila y Veliz, 2015.

6 Barends, 1977; Rodríguez, 1977; Soto, 1978; Bahamondes y col., 1979; Vila y col., 1981. 
"Esta facultad, por consiguiente, deberá subordinar su función y estructura a las exigencias de la investigación científica. De abi que el carácter de la enseñanza que se imparta también derivará de esos designios teóricos. Académicamente esto implica la voluntad de formar en determinadas disciplinas del saber antes que limitarse a informar sobre ellas. Y puesto que es inherente al Con desarrollo de conocimiento en ciencias exactas y naturales su constante proceso de elaboración y rectificación, se comprende que el problema de las condiciones de la investigación, del cultivo de la ciencia y de su enseñanza sea siempre actual y crítico".

Este texto, elaborado en el decanato de Mario Luxoro, estableció los pilares y el sentido de la formación científica en la Facultad de Ciencias, detallando además el funcionamiento de cada departamento, sus objetivos y sus mallas curriculares. Los primeros programas de formación fueron las licenciaturas en biología, química, física y matemáticas, creadas por decreto universitario el año 1966, aun cuando sus primeros estudiantes ingresaron el año 1968.

Cada departamento establecía el plan de estudio correspondiente con cursos obligatorios y electivos y el requisito para graduarse, además de haber cursado satisfactoriamente el plan de estudios, era la realización de una investigación de a lo menos seis meses, después de lo cual se debía presentar un escrito con los resultados de ésta. La duración de los programas era de cinco años. Aun cuando el Decreto Universitario del año 66 que dio origen a las licenciaturas incluía también la creación del programa de doctorado en ciencias con menciones, posteriormente se estableció el reglamento de este programa de doctorado en las mismas áreas que las licenciaturas, vale decir matemáticas, física, biología y química. Es así que el Doctorado en Biología comenzó muy tempranamente a desarrollar sus actividades y sus primeros egresados en el año 1969 fueron Cecilia Hidalgo -bioquímica, actual profesora titular de la Universidad de Chile- y Ramón Latorre, ambos Premios Nacionales de Ciencias. Cabe señalar que la Profesora Hidalgo no solo es la primera Doctora en Ciencias de la Universidad de Chile, sino que también la primera mujer en obtener el Premio Nacional en Ciencias Naturales en nuestro país.

El doctorado en el área de biología se fue diversificando en menciones, pero fue una división interna y no decretada. Aunque el peso de la cultura decía que había distintos doctorados (bioquímica, fisiología, genética, botánica y zoología), en la ley era uno solo, ya que al final todos se graduaban como doctores en ciencias con mención en biología. El primer doctorado que surgió de manera independiente fue el de Microbiología (1988), el cual se asoció con la Universidad de Santiago en el año 2000. Luego surgieron otros programas, como el de Ecología y Biología

7 Libro Naranja, pág.1, Facultad de Ciencias, 1967. 
Evolutiva y el de Biología Molecular Celular y Neurociencias, producto de la fusión de las submenciones del Doctorado en Biología Inicial. A estos programas se suma el recientemente creado Doctorado en Biotecnología Molecular del año 2012.

Con respecto al Doctorado en Química, el primer doctor fue del año 1970 y su primer graduado fue Juan Scaino Gargallo. Cabe señalar que durante los años noventa el Doctorado en Química se transformó en un doctorado interfacultad, lo que dio origen a un nuevo decreto del año 1993.

La misma situación aconteció con el programa de Doctorado en Física, que fue creado por la Facultad de Ciencias en el año 1971 y que a partir de 1988 se unió con la Facultad de Ciencias Físicas y Matemáticas, creándose las bases de la modalidad actual. Desde su creación el programa ha graduado a un total de 31 doctores, siendo los primeros graduados Miguel Lagos y Guillermo Cabrera, ambos en el año 1975. En la actualidad el primero es académico de la Universidad de Talca y el segundo, académico de la Universidad de Campinas en Brasil. El primer Doctor en Ciencias con mención en Matemáticas fue Roberto Aravire, quien se graduó el año 1989.

Con el golpe militar de 1973 empezó la intervención militar de la Universidad. En esos años duros y difíciles para todo el país la facultad no estuvo exenta de aquello; muchos académicos, estudiantes y funcionarios se marcharon por diversas razones. En el año 1974 las nuevas autoridades delegadas fusionaron las facultades de Ciencias y de Ciencias Naturales del Instituto Pedagógico, lo que implicó la incorporación de las carreras de Pedagogía en Física, Química, Matemáticas y Biología.

El intento de cerrar la facultad o más bien de fusionarla con otras era una amenaza permanente. Muchos se fueron. Paulatinamente comenzaron a volver algunos, pero otros nunca más volvieron. Sin embargo, los que se quedaron intentaron mantener esa comunidad pensante aunque quizás menos dialogante, pues la desconfianza y el miedo se instalaron en todo el país. En la formación de los alumnos esas tensiones se hicieron presentes a través de dificultades para desarrollar la investigación debido a la escasez de instrumentos y de presupuesto. Todo era mucho más complejo y los académicos debían usar mucho más la imaginación y la creatividad. Los programas de doctorado decayeron aun cuando siguieron vigentes, pero en contrapartida se conformaron los programas de magíster en el año 1978, los cuales han funcionado ininterrumpidamente.

Los años ochenta fueron determinantes en esa resistencia a mantener una facultad pequeña, pero llena de ímpetu por el saber, la experimentación y la búsqueda de respuestas en la naturaleza. Sin embargo, la Universidad de Chile en su conjunto recibía los embates de un régimen que no quería una Universidad pensante, dialogante, inclusiva y laica. El año 1981 le fueron arrebatadas las sedes regionales y el Instituto Pedagógico. La facultad fue fusionada con la Facultad de 
Ciencias Químicas (la actual Facultad de Ciencias Químicas y Farmacéuticas), pero dicha fusión duro tan solo tres años, pues conciliar dos unidades académicas tan y Química diferentes en su estructura y objetivos fue una tarea compleja. La nueva propuesta de Universidad consignaba una pequeña y disminuida y de allí que se realizó esta y otras fusiones. Sin embargo, los interventores militares no solo no entendían lo que era una universidad, sino que tampoco -al parecer- los desarrollos disciplinarios y los énfasis profesionales y/o académicos. Un papel destacado en volver la facultad a su fisonomía original le cupo al Profesor Camilo Quezada, matemático y posteriormente Decano de la misma ${ }^{8}$.

Con la recuperación de la democracia se restituyó una vida más acorde con la Universidad, que implicó mayor debate, respeto por la vida de las personas y la consideración de que el disenso era parte de la cotidianiedad y la pregunta metódica, una forma de vida. Sin embargo, los recursos para la investigación siguen siendo escasos, la institucionalidad científica, precaria, y el cultivo de la ciencia una rareza que solo se da en las aulas y laboratorios universitarios.

El deseo de hacer ciencias es la permanencia de la facultad, su sentido primigenio. La formación de investigadores de alto vuelo capaces de contribuir al desarrollo del conocimiento sigue en pie, desarrollándose cotidianamente como fue el deseo de los pioneros que la materializaron. Sin embargo, se requerían nuevas propuestas de formación y la decisión se inclinó al desarrollo de carreras profesionales.

La propuesta de formación profesional que en la actualidad posee la Facultad de Ciencias sigue basándose en los principios que planteaba el Libro Naranja:

"La ciencia en su estado actual condiciona la vida del hombre como en ninguna época de su bistoria. Las consecuencias técnicas y económicas de la actividad científica prefiguran las formas de la sociedad presente y futura"'.

Las carreras profesionales como Ingeniería en Biotecnología Molecular, Química Ambiental -ambas creadas en 1995- y Biología con Mención en Medio Ambiente (1996), cumplen con estas características, pues han sido pensadas como una formación en ciencia básica que responde a los desafíos actuales de la sociedad, vale decir, pasar a una formación aplicada, pues en ello combinan la rigurosidad de los conocimientos científicos con los aspectos productivos -en el caso de la biotecnología- o con la protección y cuidado del medio ambiente en el caso de las otras dos.

8 El profesor Quezada fue Decano entre los años 1983 y 1986 y luego entre los años 1994-2000.

9 Libro Naranja, pág.1, Facultad de Ciencias, 1967. 
Las otras carreras profesionales en las que ha incursionado la facultad son la Pedagogía en Matemáticas y Física -creada el 2005- y la Pedagogía en Biología creada el 2014. Ambas poseen un modelo innovador, pues no están centradas en una facultad, sino en colaboración con otra unidad académica, la Facultad de Filosofía y Humanidades. El trabajo colaborativo de dos o más facultades es una característica que en la Universidad de Chile es propia del posgrado, no así de la formación de pregrado. En la actualidad, estas carreras acogen a los mejores estudiantes de pedagogía del país y de esa manera la facultad contribuye al fortalecimiento de la educación en el área de las ciencias ${ }^{10}$.

El salto al desarrollo aún está pendiente como país, con una economía que sigue dependiendo de la extracción intensiva de nuestros recursos naturales y donde la contaminación ambiental hace mella. La Facultad de Ciencias ha intentado dar algunas respuestas a esos cambios, contribuyendo con la creación de carreras profesionales que puedan formar hombres y mujeres con una sólida formación científica que puedan ocuparse creativamente de los problemas que la sociedad contemporánea presenta. También se ha formado la convicción de que si la sociedad chilena en su conjunto no entiende lo que es hacer ciencia, nunca va existir un real interés en desarrollar una investigación científica como se requiere. De allí que hoy esté dentro de sus preocupaciones la alfabetización científica y lo más concreto en ello es su contribución a la formación de profesores de ciencias naturales y exactas.

Para el Dr. Maturana crear la Facultad de Ciencias fue:

“...una aventura... simplemente fue el deseo de tener alumnos que se formaran de partida como científicos, no después de recibirse de médico o de ingeniero, sino que en el proceso mismo de pronto podrían derivar en esa dirección. Yo creo que eso fue muy bueno y esto se fue ampliando, por supuesto, con los años".

La aventura lleva 50 años...

10 Los puntajes de ingreso a la pedagogía en matemáticas y física en los últimos tres años han fluctuado entre 652.6 y 797.6 el año 2013; 649.2 y 776.5 en 2014; y el 2015 entre 640.0 y 769.8, considerando el primer y último matriculado de la cohorte. Por su parte, en la primera promoción que ingresó este año a la pedagogía en biología y química los puntajes fluctuaron entre los 628.7 y 737.8 . 